\title{
Near-Infrared Emission of Nd-PSS Films
}

\author{
Marcelo C. Silva ${ }^{1}$, Fernando H. Cristovan ${ }^{2}$, Reinaldo Ruggiero ${ }^{2}$, W. O. Cruz ${ }^{2}$, and A. Marletta ${ }^{1}$ \\ ${ }^{1}$ Instituto de Física, Universidade Federal de Uberlândia, \\ Caixa Postal 593, 38400-902, Uberlândia, MG, Brazil and \\ ${ }^{2}$ Instituto de Química, Universidade Federal de Uberlândia, \\ Caixa Postal 593, 38400-902, Uberlândia, MG, Brazil
}

Received on 4 April, 2005

\begin{abstract}
We report a study of a new chemical synthetic route of polystyrene sulphonate (PSS) films doped with neodymium ion $\left(\mathrm{Nd}^{+3}\right)$ from a neodymium nitrate solution. The UV-Vis-NIR spectrum presents an intense characteristic electronic transition at $800 \mathrm{~nm}$ and the infrared spectrum presents low definition bands as a result of $\mathrm{Nd}^{3+}$ coordination with PSS molecule. Additionally, the spectrum of Nd-PSS film presents the same line shape profile of $\mathrm{Nd}^{3+}$ salt in aqueous solution. Selective luminescence spectroscopy measures shows that the incorporation of neodymium ion introduces a red shift bands and a better line shape definition in UV luminescence compared to PSS film, decreasing the interaction between PSS aromatic groups. The near-infrared emission was observed in the large spectrum region from 600 to $800 \mathrm{~nm}$; it is associated with the $\mathrm{Nd}^{3+}$ complex of PSS monomer
\end{abstract}

Keywords: Polystyrene sulphonate, Neodymium ions and synthesis route

\section{INTRODUCTION}

Optical properties of polymeric matrix doped with rare earth ions are investigated in view of the technological applications in photonic devices [1-4]. Recently, electroluminescence of polymer light emission diodes was observed from polymeric blends of poly(dioctyfluorene-cobenzothiazole) and lissamine-functionalized terphenyl-based neodymium complex[1]. This metal complex like also present an emission on near-infrared (NIR) spectral region. This protocol presents new perspectives to incorporate the rare earth elements in polymeric matrix.

In this report we have addressed a study of the chemical synthesis route of polystyrene sulphonate (PSS) films doped with neodymium ions $\left(\mathrm{Nd}^{3+}\right)$. The techniques used to this were absorption and emission spectra in the region of UVVis-NIR and FTIR, which were performed to characterize the $\mathrm{Nd}$ ion incorporation into the host matrix. The spectral range of near-infrared emission observed for $\mathrm{Nd}$ incorporated in the Nd-PSS films is from 600 to $900 \mathrm{~nm}$.

\section{EXPERIMENTAL}

The initial preceding to synthesize the Nd-PSS films was the conversion of the sodium polystyrene sulphonate (NaPSS), $\mathrm{M}_{w}=70000$, into acid form (H-PSS). Scheme 1 shows this one using an Amberlite $(I R-120$. The film processing was performed using a precursor mixing aqueous solution of $\mathrm{H}$-PSS and $\mathrm{Nd}\left(\mathrm{NO}_{3}\right)_{3}$ (neodymium nitrate) with a constant volume ratio, $6: 1(\mathrm{v}: \mathrm{v})$, respectively. The concentration of $\mathrm{Nd}\left(\mathrm{NO}_{3}\right)_{3}$ was $0.7 \mathrm{~mol} . \mathrm{L}^{-1}$ and of H-PSS was 0.15 mol.L ${ }^{-1}$. Scheme 1 shows possible steps to ionic exchange reaction between $\mathrm{H}^{+}$and $\mathrm{Nd}^{3}$. The initial solution containing $\mathrm{H}-\mathrm{PSS}$ and $\mathrm{Nd}\left(\mathrm{NO}_{3}\right)_{3}$ was stayed overnight at $5^{\circ} \mathrm{C}$ and the liquid phase was removed. Finally, the films were obtained after solvent evaporation under vacuum at room temperature.

Optical characterization of H-PSS and Nd-PSS films was

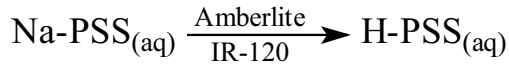

$$
\begin{aligned}
& \mathrm{H}-\mathrm{PSS}_{(\mathrm{aq})}+\mathrm{Nd}^{3+}{ }_{(\mathrm{aq})} \longrightarrow \mathrm{Nd}-\mathrm{PSS}_{(\mathrm{s})}+\mathrm{H}^{+}{ }_{(\mathrm{aq})}
\end{aligned}
$$

Scheme 1. Synthetic route of Nd-PSS films.

performed using a HACH $4000 \mathrm{U}$ spectrophotometer in UVVis-NIR region and a NEXUS 470 FTIR spectrophotometer in infrared region. The luminescence in UV-Vis-NIR region was carrier out using a Hitachi F-4500 spectrofluorimeter.

\section{RESULTS AND DISCUSSION}

Figure 1 displays the absorbance spectra in UV-Vis-NIR of H-PSS film, $\mathrm{Nd}\left(\mathrm{NO}_{3}\right)_{3}$ aqueous solution and the Nd-PSS film. We observe that the transitions present in the neodymium nitrate solution are present in the Nd-PSS film. For the spectra contain $\mathrm{Nd}$, the mean difference is the intensity ratio between the transitions ${ }^{4} \mathrm{I}_{9 / 2} \rightarrow{ }^{4} \mathrm{G}_{5 / 2}+{ }^{2} \mathrm{G}_{7 / 2}(580 \mathrm{~nm})$ and ${ }^{4} \mathrm{I}_{9 / 2} \rightarrow{ }^{4} \mathrm{~F}_{5 / 2}+{ }^{2} \mathrm{H}_{9 / 2}(800 \mathrm{~nm})$. The increase in the absorbance spectra for Nd-PSS film above $400 \mathrm{~nm}$ is due to the H-PSS matrix.

Figure 2 displays the FTIR spectra of H-PSS and Nd-PSS. In the case of H-PSS film, we observe the common bands of polystyrene sulphonate[5]: i) $1190 \mathrm{~cm}^{-1}$ - symmetric stretching of $\mathrm{S}=\mathrm{O}$, ii) $1000-1030 \mathrm{~cm}^{-1}$ - symmetric stretching of O$\mathrm{S}-\mathrm{O}$, iii) $800 \mathrm{~cm}^{-1}$ - angular deformation of $\mathrm{C}-\mathrm{H}$ of aromatic ring and iv) $3000-3900 \mathrm{~cm}^{-1}$ - symmetric stretching of $\mathrm{O}-\mathrm{H}$. For Nd-PSS film, the spectrum shows the same bands with an enlargement in all range. Sun and co-works[6] have seen a similar effect for poly(bithiazole-tetrathiapentalene) metal complex like. In this case, the result was correlated with the complex formation between the polymer and the ion. In our system seems the same coordination of $\mathrm{Nd}^{3+}$ with the sulfuric 


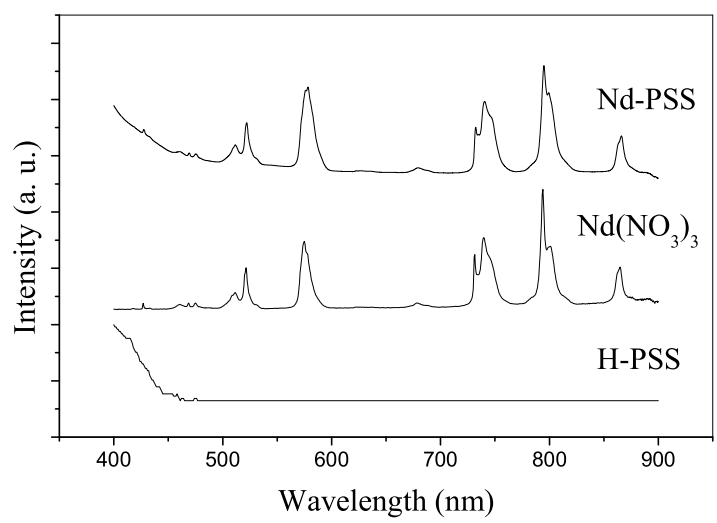

FIG. 1: Absorbance spectra of H-PSS film, aqueous solution of $\mathrm{Nd}\left(\mathrm{NO}_{3}\right)_{3}$ and Nd-PSS film in UV-Vis-NIR region.

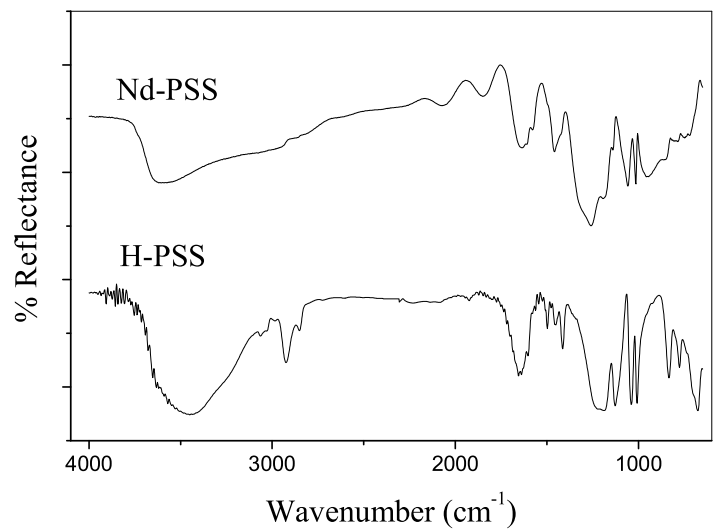

FIG. 2: FTIR spectra of Nd-PSS and H-PSS films.

groups of PSS monomers.

Figure 3 displays the PL for H-PSS and Nd-PSS films in the range between 450 and $900 \mathrm{~nm}$. For high excitation energy $(425 \mathrm{~nm})$, both spectra can be considerably approximately equal. In this case the emission is preferentially due to the PSS excimers species. When the excitation energy (550 $\mathrm{nm}$ ) decrease, the spectrum of Nd-PSS presents a consider- able red shift as compared to H-PSS film. This result is in accordance to the result obtained FTIR measurement (Fig. 2). This is more perceptive in $\mathrm{O}-\mathrm{H}$ band. Considering the complex formation of $\mathrm{Nd}^{3+}$ and the lateral chain of PSS (aromatic groups), the entanglement between adjacent polymer chains is expected to increase. Consequently, the new excimer species presents lower formation energy. This corroborates with the fact that H-PSS film is transparent in this excitation wavelength (Fig. 1).

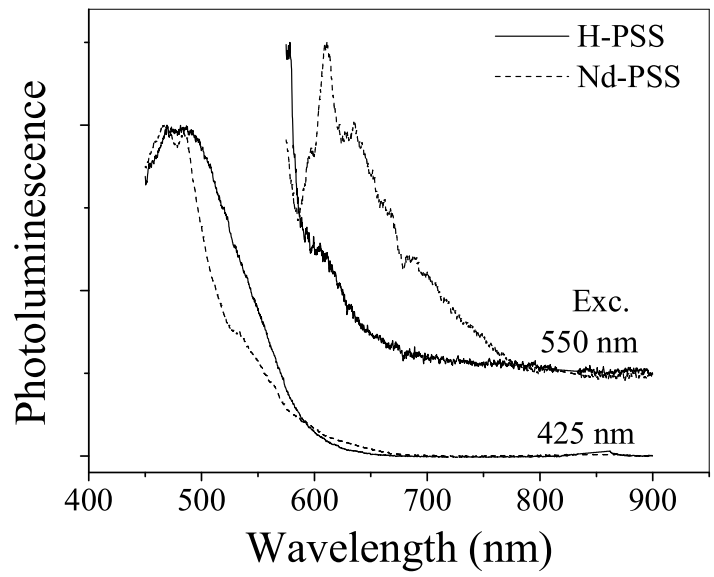

FIG. 3: Emission spectrum of H-PSS and Nd-PSS films as function of excitation wavelength.

\section{CONCLUSIONS}

In summary, it has been demonstrated that Nd-PSS films can be obtained using H-PSS and neodymium nitrate. The study of absorbance in UV-Vis-NIR and IR region show the incorporation of $\mathrm{Nd}$ ion in the polymeric matrix without the significant changes in the internal $\mathrm{Nd}^{3+}$ electronic structure. The radiative transition in near-infrared region was attributing to complex metal like.

\section{Acknowledgements}

The authors acknowledge the financial support from FAPEMIG, CNPq, CAPES and IMMP/MCT (Brazil).
[1] B. Jacquier, J. All. and Comp. 225, 15 (1995).

[2] U. Hömmerich, Ei Ei Nyein, D. S. Lee, J. Heikenfeld, A. J. Steckl, and J. M. Zavada, Mat. Sc. Eng. B 105, 91 (2003).

[3] C. Pitois, R. Vestberg, M. Rodlert, E. Malmstrom, A. Hult, and M. Lindgren, Op. Mat. 21, 499 (2003).

[4] L. H. Slooff, A. Polman, F. Cacialli, R. H. Friend, G. A. Hebbink,F. C. J. M. van Veggel, and D. N. Reinhoudt, Appl. Phys.
Lett. 78(15), 2122 (2001).

[5] D. L. Paiva, G. M. Lampman, and G. S. Kris, Introduction to Spectroscopy, Saunders College, $2^{a}$ ed., USA, 1996.

[6] W. Sun, L. Jiang, J. Weng, B. He, D. Cen, and Z. Shen, Reac. Func. Pol. 55, 249 (2003). 